\title{
Targeted Theranostic Approach for Glioma Using Dendrimer-Based Curcumin Nanoparticle \\ Gamage $\mathrm{NH}^{1}$, Jing $\mathrm{Li}^{2}$, Worsham $\mathrm{MJ}^{1}$ and Ali $\mathrm{MM}^{1 *}$
}

${ }^{1}$ Department of Neurology, Henry Ford Hospital, Detroit, MI 48202, USA

${ }^{2}$ Department of Oncology, Wayne State University School of Medicine, Detroit, MI 48202, USA

\begin{abstract}
The delivery of anti-cancer agents to brain tumors represent a challenge because the blood-brain tumor barrier (BBTB) effectively limits the delivery of many agents. A new generation 3 (G3) dendrimer-based curcumin (Curc) conjugate was synthesized. The synthesized G3-Curc conjugate demonstrated full solubility in aqueous media. The in vitro study revealed that G3-Curc nanoparticles were internalized into glioma U-251 cells. Systemic delivery of G3-Curc conjugate led to preferentially accumulation in an orthotopic preclinical glioma model minimizing systemic toxic effect. Multicolor microscopy images of the tumor tissue showed that G3-Curc particles were internalized inside tumor cells selectively and further localized within nuclei. Enhanced bioavailability of G3-Curc conjugate was also observed with improved therapeutic efficacy against different cancers cells.
\end{abstract}

Keywords: Dendritic curcumin conjugate; Glioma specific agent; Optical imaging; Nanotheranostic

\section{Introduction}

Glioblastoma (GBM) is one of the most aggressive brain tumors, and typically exhibits hyper vascularity and marked heterogeneity. GBM constitutes a major clinical problem because of its high rate of recurrence and aggressive phenotype leading to extremely poor prognosis [1]. Accordingly, it is often impossible to resect $100 \%$ of tumor mass during surgery and the left over tumor cells might become more aggressive and resistant to future therapies. Although is growing evidence that chemotherapy plays an increasingly significant role in treatment of malignant gliomas, the benefit in survival improvement is not satisfactory [1-3]. Mortality due to brain cancer has essentially remained unchanged in the last three decades. Therefore, it is important to explore and identify more efficient therapeutic strategies across the blood-brain tumor barrier (BBTB), coupled with an ability to image the true extent of drug delivery to the tumor for the treatment of malignant gliomas. A number of preclinical and clinical studies suggest that natural compounds such as curcumin may represent useful additions to therapeutic regimens of cancer patients [4-6].

Curcumin (Curc), a yellow pigment in the spice turmeric (Curcuma longa), has been reported for its potential chemopreventive and chemotherapeutic activity by influencing various aspects, such as cell cycle arrest, differentiation, and apoptosis in a series of cancers [7-14]. In addition, Curc potently inhibited proliferation, migration and invasion of GBM cells in in- vitro studies [15]. In human prostate cancer LNCaP cells, Curc reversed the CpG methylation of the promoter region of Neurog1 with restoration of its mRNA and protein expression [16]. Nevertheless, a major criticism of Curc has been the apparent poor systemic bioavailability in in-vivo animal models. The latter indicates poor relevance for clinical translation even when patients were given up to 8-10 grams of the free drug orally each day [12]. In addition, systemic delivery of Curc leads to non-specific distribution throughout the body [17]. It is reasonable to explore novel formulations of Curc that overcome the limitations mentioned above. Many attempts have been made to improve the solubility of the highly water insoluble Curc using various types of nanoparticle (NPs), such as polymer NPs, micelles, liposomes, nano-/microemulsions, nanogels, solid lipid NPs, polymer conjugates, self-assemblies, and so on $[12,17,18]$. These NPs have been used for the delivery of an active form of Curc to tumors [17].
While these long-circulating nanocarriers significantly increase tumor localization of the payload, some limitations exist. First, the targeting effect is highly dependent on the degree of tumor vascularization and angiogenesis. The porosity and pore size of tumor vessels vary with the type and status of the tumor; thus, the passive targeting effect may not be always achieved in all tumors. For example, a recent study evaluated the influence of nanoparticle size on BBTB permeability and showed that gadolinium chelated dendrimer nanoparticles with core sizes of $<12 \mathrm{~nm}$ were able to permeate the BBTB, whereas larger nanoparticles were hindered [19]. Thus, progress toward the effective clinical treatment of GBM has been hampered due to ineffective drug delivery across the BBTB, combined with an inability to image the true extent of drug delivery to the tumor.

We have recently developed dendrimer-based paramagnetic nanoparticles that are detected by standard magnetic resonance (MR) relaxivity methods [20,21] or new MRI methods based on PARAmagnetic Chemical Exchange Saturation Transfer (PARACEST) $[22,23]$. We have demonstrated that a generation 5 (G5) PAMAM dendrimer decorated with PARACEST agents and fluorophore preferentially accumulated in an orthotopic pre-clinical U87 glioma due to the enhanced permeability and retention effect (EPR). This dendritic conjugate is an attractive foundation for the development of a broadly useful platform for both targeted brain tumor drug delivery and non-invasive molecular imaging. Here, we report a new Curcconjugated generation 3 (G3) dendrimer to improve in vivo systemic bioavailability and deliver drug selectively into brain tumors with a compromised BBTB, thus improving its diagnostic imaging potential (i.e. theranostic) as well as minimizing drug-related systemic toxicity. This water soluble G3-Curc prodrug is a promising new formulation

*Corresponding author: Meser M. Ali, One Ford Place, 2F, Department of Neurology, Henry Ford Hospital, Detroit, MI 48202, USA, Tel: +1- 313-874-4479; Fax: +1-313-874-4494; E-mail: mali8@hfhs.org

Received July 15, 2016; Accepted August 05, 2016; Published August 12, 2016

Citation: Gamage NH, Li J, Worsham MJ, Ali MM (2016) Targeted Theranostic Approach for Glioma Using Dendrimer-Based Curcumin Nanoparticle. J Nanomed Nanotechnol 7: 393. doi: 10.4172/2157-7439.1000393

Copyright: @ 2016 Gamage NH, et al. This is an open-access article distributed under the terms of the Creative Commons Attribution License, which permits unrestricted use, distribution, and reproduction in any medium, provided the original author and source are credited. 
that overcomes a major impediment critical for clinical translation of Curc to cancer patients by improving systemic bioavailability, and by extension, therapeutic efficacy.

\section{Materials and Methods}

\section{Materials}

All reagents used were purchased from Sigma Aldrich (St. Louis, MO) unless otherwise stated. G3-PAMAM succinamic acid (10 wt.\%) in methanolwasobtained from Dendritech (Midland,MI) anditwasdriedin vacuo prior to use. 4-Dimethylaminopyridine (99\%) Dimethylsulfoxide anhydrous ( $\geq 99.9 \%), \quad \mathrm{N}, \mathrm{N}^{\prime}$-dicyclohexylcarbodiimide $(99 \%)$ and Sodium bicarbonate (100\%) were purchased from Sigma Aldrich and were used without further purifications. Sodium hydroxide (100\%) and 2-Methylbutane (95\%) were purchased from Fisher Scientific and were also used without further purification. Dendrimeric curcumin conjugates were purified by repeated ultrafiltration with deionized water using appropriate molecular weight cut-offs (Millipore's Amicon Ultra centrifugal filters). ${ }^{1} \mathrm{H}$ - NMR spectra were obtained using an Agilent 600 NMR spectrophotometer operating at $599.77 \mathrm{MHz}$. Deuterated DMSO(DMSO- $\mathrm{d}_{6}$ ) and Deuterated $\mathrm{D}_{2} \mathrm{O}$ were used as NMR solvents and Chemical shifts $(\delta)$ are given in ppm with reference to the internal standard Tetramethylsilane (TMS, $\delta=0 \mathrm{ppm}$ ). The molecular weights of the conjugates were analyzed using a Micromass M@LDI-R or Applied Biosystems DE Maldi-TOF spectrometer.

\section{MRI method}

An isotropic 3D FIESTA MR image was acquired to locate the U251 glioma in the rat model using $3 \mathrm{~T}$ (GE, excite) clinical MRI system fitted with dedicated small animal coil (Litzcage small animal imaging system, Doty Scientific Inc, Columbia, SC) [23]. The FIESTA images were obtained with the following parameters: TR: $11.4 \mathrm{msec}$;E: 5.61 msec; 0.3 mm slice thickness; 200 x 200 matrix; 300 x $300 \mu \mathrm{m}$ in-plane resolution; $60 \times 60 \mathrm{~mm}$ field of view; 2 averages. To prepare for the MRI exam, a rat was anesthetized with $1.5-2.0 \%$ isoflurane delivered in $2 \mathrm{~L} / \mathrm{min}$ oxygen gas ventilation, and a catheter was inserted into the tail vein to facilitate the injection of the MRI contrast agent. The rat was secured in a customized MRI-compatible cradle to prevent injury during the MRI exam, and probes for monitoring rectal temperature and respiration was connected to the rat. The tumor was visualized by the intravenous injection of clinically used Gd-DTPA $(0.1 \mathrm{mmol}$ based on $\mathrm{Gd} / \mathrm{kg}$ concentration).

\section{Optical imaging}

After in vivo imaging, the rat was euthanized using $\mathrm{CO}_{2}$ asphyxiation and perfused by intracardiac injection of $100 \mathrm{~mL}$ PBS followed by $3 \%$ paraformaldehyde, and then kept in a solution of 3\% sucrose and $3 \%$ paraformaldehyde during storage [22]. An ex vivo fluorescence images of the whole brain and the major organs were acquired using a Kodak Carestream Multispectral Imaging System (Carestream, USA). To obtain an optimal emission image devoid of non-specific fluorescent emission, fluorescence images were acquired using multiple excitation wavelength filters ranging from 400 to $480 \mathrm{~nm}$ and a single emission wavelength filter of $600 \mathrm{~nm}$. Spectral profiles were created using spectral analysis software (Carestream) and subtracted optimal images were obtained. For each optical image set, an X-ray image was also obtained to validate the anatomic location of the tumor as well as major organs (the Fluorescence and X-ray images were overlayed). The brain was snap-frozen and cut into 6-8 $\mu \mathrm{m}$ thick sections for further analysis with fluorescence microscopy. For fluorescent microscopic detection of curcumin conjugated to G3, a proper excitation and emission filter was used. Nuclei were visualized with DAPI (Sigma), using standard histological staining procedures as recommended by the suppliers of reagents.

\section{LC-MS/MS method for determination of curcumin in serum}

The concentrations of curcumin in serum were determined using a high-performance liquid chromatographic method with mass spectrometric detection (LC-MS/MS). To $100 \mu \mathrm{l}$ of plasma, $200 \mu \mathrm{l}$ methanol containing $2 \%$ formic acid was added, and the mixture was vortex-mixed for 1 minutes, centrifuged at 14000 RPM for 5 minutes. Zileuton was used as internal standard. The supernatant was collected, and a volume of $10 \mu \mathrm{l}$ was injected into the HPLC.

Chromatographic analysis was performed using a Waters Model 2695 separations system (Milford, MA, USA). Separation of the analytes from potentially interfering material was achieved using Waters Xterra MS C18 column ( 50 x $2.1 \mathrm{~mm}$ i.d., $3.5 \mu \mathrm{m})$ protected by a Waters Xterra MS C18 guard column $(3.5 \mu \mathrm{m}, 10 \times 2.1 \mathrm{~mm}$ i.d $)$. The mobile phase used for the chromatographic separation was composed of methanol $/ 0.45 \%$ formic acid in water $(70: 30, \mathrm{v} / \mathrm{v})$, and was delivered isocratically at a flow rate of $0.2 \mathrm{~mL} / \mathrm{min}$. The column effluent was monitored using a Waters Quattro Micro $^{\text {ma }}$ triple quadrupole massspectrometric detector (Milford, MA, USA). The instrument was equipped with an electrospray ionization source, and controlled by the Masslynx version 4.1 software. The samples were analyzed using an electrospray probe in the negative ionization mode operating at a cone voltage of $24 \mathrm{~V}$ for curcumin and in the positive ionization mode at a cone voltage of $13 \mathrm{~V}$ for internal standard zileuton. Samples were introduced into the ionization source through a heated nebulized probe $\left(350^{\circ} \mathrm{C}\right)$. The spectrometer was programmed to allow the $[\mathrm{MH}]^{+}$ ion of curcumin at $\mathrm{m} / \mathrm{z} 367.0$ and zileuton at $\mathrm{m} / \mathrm{z} 237.0$ to pass through the first quadrupole (Q1) and into the collision cell (Q2). The collision energy was set at 22 and $19 \mathrm{eV}$ for curcumin and zileuton, respectively. The product ions for curcumin $(\mathrm{m} / z 149.0)$ and zileuton $(\mathrm{m} / z$ 161.0) were monitored through the third quadrupole (Q3). Argon was used as collision gas, and the dwell time per channel was $0.2 \mathrm{sec}$ for data collection. The linear calibration curve was set at curcumin serum concentrations ranging from 10 to $10,000 \mathrm{ng} / \mathrm{ml}$. The intra- and interday accuracy and precision for QC samples were within $15 \%$.

\section{Synthesis of G3-PAMAM-(Succinimic acid)-Curcumin ${ }_{24}$ conjugate}

To a $100 \mathrm{~mL}$ round bottom flask, was added G3-PAMAM(succinimic acid) ${ }_{32}(0.27 \mathrm{~g}, 0.027 \mathrm{mmol}, 1$ equiv.) and it was dissolved in DMSO $(10 \mathrm{~mL})$. To a separate $50 \mathrm{~mL}$ centrifuge tube, was added curcumin ( $0.3136 \mathrm{~g}, 0.85 \mathrm{mmol}, 32$ equiv.) and it was dissolved in DMSO $(10 \mathrm{~mL})$. To the solution of G3-PAMAM-(succinimic acid) $)_{32}$ in DMSO was added the solution of curcumin and the centrifuge tube was rinsed with portions of DMSO $(2 \times 5 \mathrm{~mL})$, which was also added to the reaction mixture. Then, to the reaction were added 4-dimethylaminopyridine $(1.0 \mathrm{mg})$ and $\mathrm{N}, \mathrm{N}$-Dicyclohexylcarbodiimide $(0.1756 \mathrm{~g}, 0.8512 \mathrm{mmol}$, 32 equiv.) and the reaction was left to stir for $24 \mathrm{~h}$ at room temperature. After transferring the reaction to a $500 \mathrm{~mL}$ Erlenmeyer flask, it was diluted by the addition of distilled water $(300 \mathrm{~mL})$ and was allowed to stir at room temperature for $0.5 \mathrm{~h}$. Then, the reaction mixture was filtered multiple times until a clear solution was observed. Finally, the filtrate was diafiltered using Amicon ${ }^{\circ}$ Ultra centrifugal filter $(3,000$ MWCO, regenerated cellulose) and was washed with distilled water (3 times) under the same conditions. Then, the sample was pre-frozen in dry ice cooled 2-methylbutane and was freeze dried on the Freeze drier. 
Finally, $0.5108 \mathrm{~g}$ of yellowish orange product was obtained. Maldi-TOF (Supporting information Figure S1) m/z: 19005 g.

\section{Result and Discussion}

Curcumin (Curc) was conjugated with G3-succinamic acid dendrimer $\left(\mathrm{G} 3-\mathrm{COOH}_{32}\right)$ through DMP (4-dimethylaminopyridine)/ DCC (dicyclohexylcarbodiimide) coupling method. The conjugate, G3-Curc (Figure 1A) was purified by diafiltration using C-3 membrane filter (MW cutoff size $>3,000 \mathrm{~g}$ ). G3-Curc was characterized by ${ }^{1} \mathrm{H}$ NMR, Uv-visible and maldi-tof mass spectrometric methods. ${ }^{1} \mathrm{H}$ NMR spectrum (Figure 1B) showed the multiplets between $\Delta 1.0$ and $4.0 \mathrm{ppm}$ corresponding to the presence of $-\mathrm{CH}$ and $-\mathrm{CH}_{2}$ protons from Curc and PAMAM G3-succinamic acid [24,25]. On the other hand, several peaks between $\Delta 6.6$ and $8.4 \mathrm{ppm}$ showed the presence of hydrogen atoms on the phenyl ring in Curc [25]. Due to the overlapping of the multiple peaks in the NMR spectrum, the complete characterization of the protons was not achieved. Using maldi-tof mass spectroscopy the number of Curc molecules per dendrimer was estimated. G3succinamic acid $\left(\mathrm{G} 3-\mathrm{COOH}_{32}\right)$ has a mass of $10,109 \mathrm{~g} / \mathrm{mole}$ and G3Curc conjugate was shown to have a mass of $19,005 \mathrm{~g} / \mathrm{mole}$ (Figure S1). Since Curc has an approximate mass of $368.38 \mathrm{~g}$, the conjugate was shown in Figure 1A to contain 24 Curc molecules per dendrimer corresponding to a conjugation yield of $75 \%$. The G3-Curc polymer conjugate (Figure 1A) was highly water soluble while natural free Curc was completely water insoluble as shown in Figure S2. Therefore, we have succeeded in conjugating a water insoluble Curc with highsolubility G3 polymers that bear an additional advantage as a prodrug with enhanced aqueous solubility. As a water soluble prodrug, it should further increase the bioavailability of the drug and therefore have the potential to enhance its cytotoxic effect. The absorption spectra of Curc, G3-succinamic acid and G3-Curc conjugate (in $\mathrm{H}_{2} \mathrm{O}$ ) is shown in Figure S3. Only G3-Curc showed an absorption maxima $\left(\lambda_{\max }\right)$ around $435 \mathrm{~nm}$. However, in dimethyl sulfoxide (DMSO) Curc and G3-Curc also showed absorption maxima at $435 \mathrm{~nm}$ (Figure S4). Therefore, conjugation of Curc to G3 dendrimer did not shift the absorption maxima of free Curc. Free Curc (in DMSO) was used to generate a standard concentration curve for the estimation of Curc content in G3-

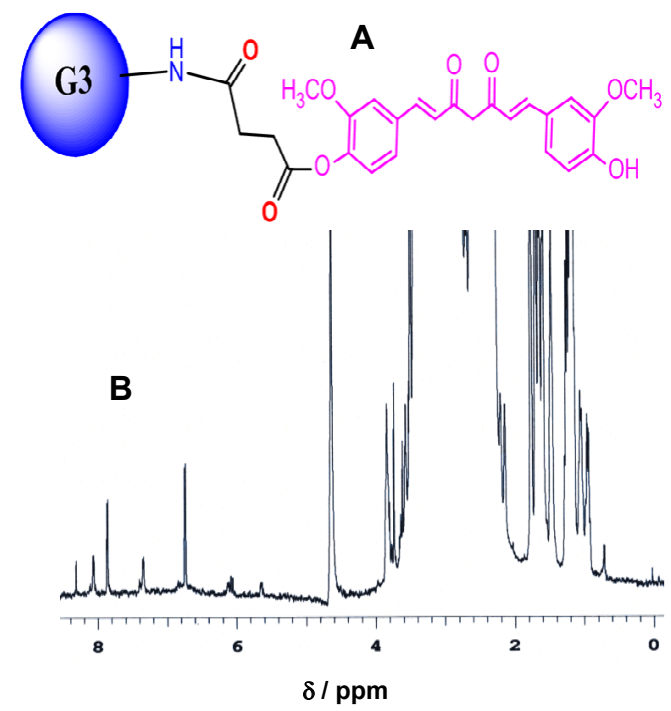

Figure 1: Schematic view of curcumin conjugated with a G3 PAMAM succinamic acid dendrimer conjugate (A). ${ }^{1} \mathrm{H}$ NMR spectrum of G3-(Curc) in $\mathrm{D}_{2} \mathrm{O}(\mathrm{B})$.
Curc conjugate. The content of Curc in the conjugate was estimated from the absorption spectroscopic method which was also in good agreement with the result based on maldi-tof.

To study cellular internalization of G3-Curc, we added G3-Curc to the glioma U251 cells and incubated for $3 \mathrm{~h}$. Then we washed the cells at pH 7.4 to remove any reversibly bound G3-Curc. The cellular uptake of G3-Curc was significantly higher as clearly demonstrated in Figure 2. The location of the green fluorescence from Curc indicates perinuclear and cytosolic distribution of G3-Curc. In contrast Curc was insoluble in water and no intracellular internalization was observed. Therefore, Curc was mainly transported into the cells by nanoparticles and localized in both the nuclei and the cytoplasm (Figure 2).

All animal experiments were approved by Institutional Animals Care and User Committee (IACUC). We determined serum pharmacokinetics and tissue distribution of the G3-Curc. In order to perform bioavailability of G3-Curc in mice, the mice were randomly assigned to either G3-Curc or Curc group. G3-Curc (100 $\mathrm{mg} / \mathrm{kg}$ equivalent to Curc in water) was administered to mice by intravenously. The mice were sacrificed at $0.5,1.0,1.5,3.0$ and 4.0 hours later by euthanasia, the blood was collected by heart puncture, and the serum was separated by centrifugation at $14000 \mathrm{rpm}$ for $2 \mathrm{~min}$. HPLC profiles of serum were depicted in Figure S5. 3 mice were used for each time point. A maximum serum concentration of $90 \mathrm{ng}$ of Curc per $\mathrm{mL}$ was detected at $0.50 \mathrm{~h}$ after intravenous administration of G3Curc. However, curcumin levels in the serum reported was as "total" curcumin (free drug plus drug released from G3-Curc conjugate) per milliliter of serum. Many preclinical and clinical studies in mice, rats and humans revealed a low bioavailability of curcumin [26]. Although 10 or $12 \mathrm{~g} / \mathrm{ml}$ of curcumin administered orally in humans showed curcumin levels in serum to be approximately $50 \mathrm{ng} / \mathrm{ml}$, this resulted in a minimum availability of curcumin in the blood circulation to achieve its therapeutic effects [27]. Therefore, conjugation of Curc to a G3 dendrimer resulted an improved bioavailibity of Curc.

Orthotopic human glioma was created in nude rats by implanting $4 \times 10^{5}$ human U251 malignant glioma cells according to our recent published method $[22,28]$. A T1 weighted multislice coronal sequence (TR: $11.4 \mathrm{msec}$; TE: $5.61 \mathrm{msec} ; 0.3 \mathrm{~mm}$ slice thickness; 200 x 200 matrix; $300 \times 300 \mu \mathrm{m}$ in-plane resolution; $60 \times 60 \mathrm{~mm}$ field of view; 2 averages) was run with Gd-DTPA injection in order to locate the tumor (Figure 3a). Immediately after acquisition of the MRI data was completed, G3Curc (20 $\mu \mathrm{M}$ based on Curc concentration) was injected intravenously. The following day, the animal was sacrificed and the organs were collected by perfusion for ex-vivo fluorescence imaging. A higher fluorescence signal is detected in the tumor (Figure $3 \mathrm{~b}$ ). We achieved high contrast ratio (8-10), which suggests that G3-Curc mostly
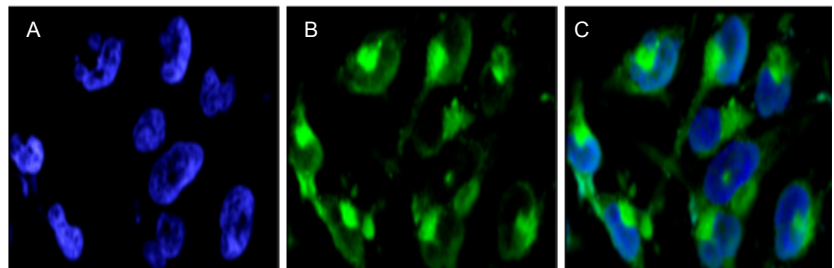

Figure 2: Fluorescent microscopic images of glioma (U251) cells incubated with G3-Cur ( $6 \mu \mathrm{M}$ based on Curc concentration) for 3 hours. Intracellular uptake was visualized by green fluorescence of Curc conjugated to G3 succinamic acid dendrimer. Nuclei were visualized with DAPI (bluer accumulation) in panel A. Green fluorescence from G3-Curc nanoparticles is shown in panel B. Panel $\mathrm{C}$ depicts merged images of Curc and DAPI fluorescence. 

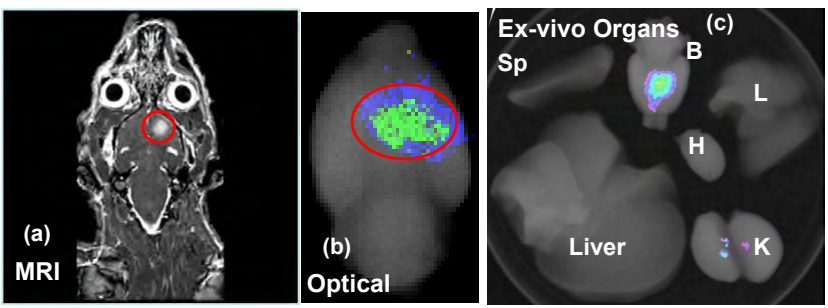

Figure 3: The coronal MRI image shows the location of U-251 glioma tumor (a). Optical image obtained under simultaneous white light and filtered (420-480 nm) excitation detected with the emission filter set at $600 \mathrm{~nm}$ demonstrating fluorescence in the rat glioma (b). Distribution of G3-Curc in organs (c). Fluorescence images of kidney $(K)$, spleen $(\mathrm{Sp})$, liver, heart $(\mathrm{H})$ and lung $(\mathrm{L})$ and brain $(\mathrm{B})$ collected from a rat bearing a tumor on the next day after injection. G3-Curc selectively accumulated at the glioma tumor site but not contralateral brain.
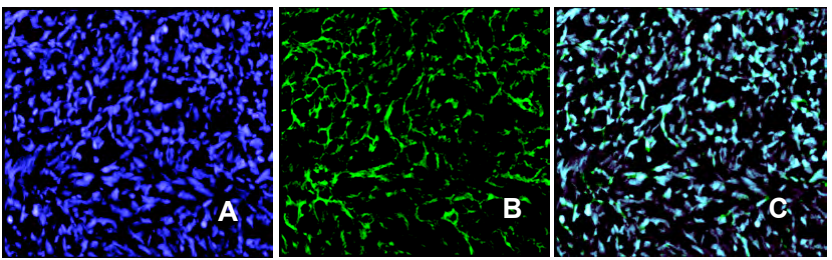

Figure 4: Microscopic fluorescence imaging of frozen tissue section from tumo (20X). DAPI (blue) staining illustrates nuclei (A). Strong, selective distribution of the G3-Curc nanoparticles (green) were visualized in tumor tissue (B). Overlays of fluorescence from the G3-Curc (green) and DAPI are shown in panel C. A high-resolution view showed accumulation of the G3-Curc within tumor cell nuclei validated by DAPI staining for viable cellularity.

accumulates in the tumor. Thus, G3-Curc nanoparticles showed more accumulation in tumor and/or less renal uptake (Figure 3c-K), likely due to small particle size, optimal surface charge, and hydrophilicity in blood. It has been demonstrated that after oral administration of $400 \mathrm{mg}$ of curcumin to rats only traces of unchanged drug were found in the liver and kidney. At $30 \mathrm{~min}, 90 \%$ of curcumin was found in the stomach and small intestine, but only $1 \%$ was present at $24 \mathrm{~h} \mathrm{[29].}$ While Curc has shown a wide range of non-specific in vivo distribution [12], G3-Curc preferentially accumulated at the tumor site.

For further fluorescence microscopy analysis, the U-251 tumor was snap-frozen and cut into 6-8 $\mu \mathrm{m}$ thick sections. The specificity of G3-Curc was further investigated with ex vivo multicolor fluorescence microscopy, which showed accumulation of the particles in glioma tumor tissue selective for nuclear distribution (Figure 4). Therefore, conjugation of Curc to G3 carrier improved bioavailability and tumor targeting. Importantly, tumor selective biodistribution (Figure 3) of G3-Cur suggests minimum drug induced side effects in normal tissue. To the best of our knowledge, this is the first example of curcuminbased nanoparticle that can cross BBTB and accumulated into U-251 glioma tumor selectively.

In order to examine our hypothesis that water soluble G3-Curc improves therapeutic efficacy, we evaluated in vitro cytotoxicity of G3Curc against a series of cancer cell lines by the MTT assay. After 24 hours incubation of G3-Curc, cytotoxicity of G3-Curc and free Curc were evaluated in MDA-MB-231 breast cancer cells, U-251 human malignant glioma, and squamous head and neck cancer (HNSCC) cells (HPV [30] negative and HPV positive cells). In vitro studies proved that the cytotoxicity of G3-Curc conjugate was more effective against different types of cancer cells in a dose-dependent manner (Figure 5). In

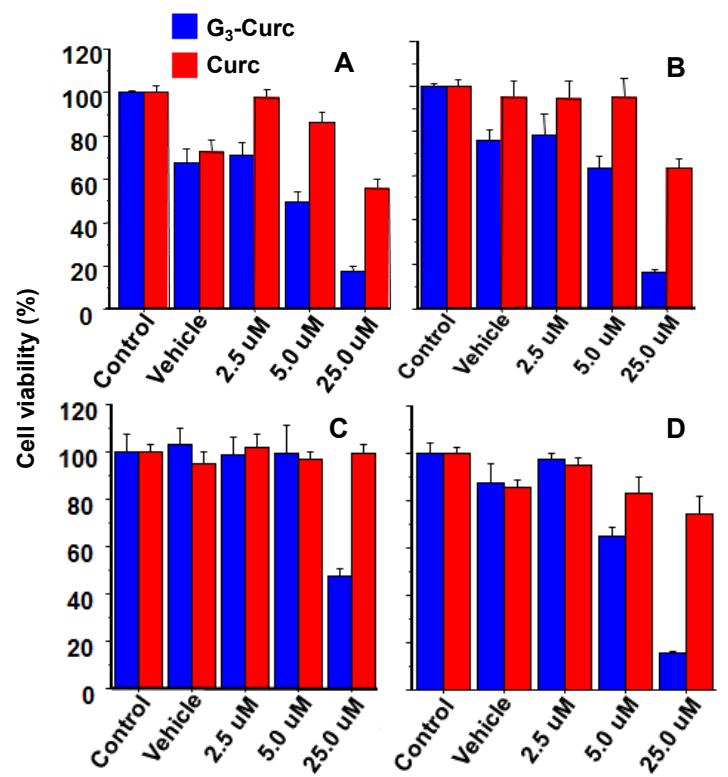

Figure 5: Cytotoxicity of G3-Curc ( $\square)$ and free Curc ( $\square)$ was evaluated by MTT assay against U-251 human malignant glioma (A), MDA-MB-231 breast cancer (B), squamous head and neck cancer (HNSCC) cells, HPV+ (C) and HPV- (D) cells. In vitro studies proved that the cytotoxicity of G3-Curc conjugate was more effective against different types of cancer cells in a dose-dependent manner.

addition, G3-Curc conjugate showed lower $\mathrm{IC}_{50}$ than that of free Curc. While small molecule chemotherapeutic drugs are insensitive against HPV negative cells [30], interestingly G3-Curc was more sensitive against HPV negative cells than that of HPV positive cells (Figure 5C and 5D). The present results suggest that G3-Curc conjugate could be a potentially useful chemotherapeutic formulation for different types of cancer therapy including drug insensitive HPV negative cells.

\section{Conclusion}

In this study, we have developed a new dendrimer-based curcumin conjugate which improves water solubility and the bioavailability of curcumin. Ex-vivo fluorescence imaging showed tumor specific distribution of the G3-Curc conjugate avoiding other major organs. Additionally, ex vivo fluorescence imaging and fluorescent microscopy of the tumor tissue indicates specificity for nuclear distribution of G3Curc. Therefore, G3-Curc conjugate is a promising therapeutic agent for the treatment of human diseases.

\section{Acknowledgements}

The authors acknowldege research support from the National Institutes of Health (NIH) grant RO1CA206190 to MMA.

\section{Disclosure}

The authors report no conflicts of interest in this work.

\section{References}

1. Huse JT, Holland EC (2010) Targeting brain cancer: advances in the molecular pathology of malignant glioma and medulloblastoma. Nature reviews Cancer 10: $319-331$.

2. Fine HA, Dear KB, Loeffler JS, Black PM, Canellos GP (1993) Meta-analysis of radiation therapy with and without adjuvant chemotherapy for malignant gliomas in adults. Cancer 71: 2585-2597.

3. Stewart LA (2002) Chemotherapy in adult high-grade glioma: a systematic review and meta-analysis of individual patient data from 12 randomised trials. Lancet 359: 1011-1018. 
Citation: Gamage NH, Li J, Worsham MJ, Ali MM (2016) Targeted Theranostic Approach for Glioma Using Dendrimer-Based Curcumin Nanoparticle. J Nanomed Nanotechnol 7: 393. doi: 10.4172/2157-7439.1000393

4. Bar-Sela G, Epelbaum R, Schaffer M (2010) Curcumin as an anti-cancer agent: review of the gap between basic and clinical applications. Current medicina chemistry 17: 190-197.

5. Baumeister P, Reiter M, Harréus U (2012) Curcumin and other polyphenolic compounds in head and neck cancer chemoprevention. Oxid Med Cell Longev: 902716 .

6. Chauhan DP (2002) Chemotherapeutic potential of curcumin for colorectal cancer. Curr Pharm Des 8: 1695-1706.

7. Cho YA, Lee W, Choi JS (2012) Effects of curcumin on the pharmacokinetics of tamoxifen and its active metabolite, 4-hydroxytamoxifen, in rats: possible role of CYP3A4 and P-glycoprotein inhibition by curcumin. Pharmazie 67: 124-130.

8. Choi BH, Kim CG, Bae YS, Lim Y, Lee YH, et al. (2008) p21 Waf1/Cip1 expression by curcumin in U-87MG human glioma cells: role of early growth response-1 expression. Cancer Res 68: 1369-1377.

9. Choi BH, Kim CG, Lim Y, Shin SY, Lee YH (2008) Curcumin down-regulates the multidrug-resistance mdr1b gene by inhibiting the PI3K/Akt/NF kappa B pathway. Cancer Lett 259: 111-118.

10. Choi H, Chun YS, Kim SW, Kim MS, Park JW (2006) Curcumin inhibits hypoxiainducible factor-1 by degrading aryl hydrocarbon receptor nuclear translocator: a mechanism of tumor growth inhibition. Mol Pharmacol 70: 1664-1671.

11. Choudhuri T, Pal S, Das T, Sa G (2005) Curcumin selectively induces apoptosis in deregulated cyclin D1-expressed cells at G2 phase of cell cycle in a p53dependent manner. The Journal of biological chemistry 280: 20059-20068.

12. Yallapu MM, Jaggi M, Chauhan SC (2012) Curcumin nanoformulations: a future nanomedicine for cancer. Drug Discov Today 17: 71-80.

13. Yang CL, Liu YY, Ma YG (2012) Curcumin blocks small cell lung cancer cells migration, invasion, angiogenesis, cell cycle and neoplasia through Janus kinase-STAT3 signalling pathway. PloS one 7: e37960.

14. Yang CW, Chang CL, Lee HC, Chi CW, Pan JP, et al. (2012) Curcumin induces the apoptosis of human monocytic leukemia THP-1 cells via the activation of JNK/ERK pathways. BMC Complement Altern Med 12: 22.

15. Senft C, Polacin M, Priester M, Seifert V, Kogel D, Weissenberger J (2010) The nontoxic natural compound Curcumin exerts anti-proliferative, anti-migratory, and anti-invasive properties against malignant gliomas. BMC cancer 10: 491.

16. Bhojwani D, Pei D, Sandlund JT, Jeha S, Riberio RC, et al. (2012) ETV6RUNX1-positive childhood acute lymphoblastic leukemia: improved outcome with contemporary therapy. Leukemia 26: 265-270.

17. Yallapu MM, Othman SF, Curtis ET, Bauer NA, Chauhan N, et al. (2012) Curcumin-loaded magnetic nanoparticles for breast cancer therapeutics and imaging applications. International journal of Nanomedicine 7: 1761-1779.
18. Yallapu MM, Othman SF, Curtis ET, Gupta BK, Jaggi M, et al. (2011) Multifunctional magnetic nanoparticles for magnetic resonance imaging and cancer therapy. Biomaterials 32: 1890-1905.

19. Sarin H, Kanevsky AS, Wu H, Sousa AA, Wilson CM, et al. (2009) Physiologic upper limit of pore size in the blood-tumor barrier of malignant solid tumors. Journal of translational medicine 7: 51.

20. Ali MM, Woods M, Caravan P, Opina AC, Spiller M, et al. (2008) Synthesis and relaxometric studies of a dendrimer-based $\mathrm{pH}$-responsive $\mathrm{MRI}$ contrast agent. Chemistry 14: 7250-7258.

21. Ali MMB, Bagher-Ebadian H, Janic B, Knight RA, Ewing JR et al. (2011) A new R2/R1 Ratiometric Method to Measure $\mathrm{pH}$ with a Dendrimer-based $\mathrm{pH}$ Responsive MRI Contrast Agent. Proc Intl Soc Mag Reson Med 19: 1688.

22. Ali MM, Janic B, Babajani-Feremi A, Varma NR, Iskander AS, et al. (2010) Changes in vascular permeability and expression of different angiogenic factors following anti-angiogenic treatment in rat glioma. PLoS One 5: e8727.

23. Ali MM, Bhuiyan MP, Janic B, Varma NR, Mikkelsen T, et al. (2012) A nanosized PARACEST-fluorescence imaging contrast agent facilitates and validates in vivo CEST MRI detection of glioma. Nanomedicine (Lond) 7: 1827-1837.

24. Lalwani S, Venditto VJ, Chouai A, Rivera GE, Shaunak S, et al. (2009) Electrophoretic Behavior of Anionic Triazine and PAMAM Dendrimers: Methods for Improving Resolution and Assessing Purity Using Capillary Electrophoresis. Macromolecules. 42: 3152-3161.

25. Shi W, Dolai S, Rizk S, Hussain A, Tariq H, et al. (2007) Synthesis of monofunctional curcumin derivatives, clicked curcumin dimer, and a PAMAM dendrimer curcumin conjugate for therapeutic applications. Organic letters 9 : 5461-5464.

26. Goel A, Kunnumakkara AB, Aggarwal BB (2008) Curcumin as "Curecumin": from kitchen to clinic. Biochem Pharmacol 75: 787-809.

27. Lao CD, Ruffin MT 4th, Normolle D, Heath DD, Murray SI, et al. (2006) Dose escalation of a curcuminoid formulation. BMC Complement Altern Med 6: 10.

28. Ali MM, Kumar S, Shankar A, Varma NR, Iskander AS, et al. (2013) Effects of tyrosine kinase inhibitors and CXCR4 antagonist on tumor growth and angiogenesis in rat glioma model: MRI and protein analysis study. Translational oncology 6: 660-669.

29. Ravindranath V, Chandrasekhara N (1980) Absorption and tissue distribution of curcumin in rats. Toxicology 16: 259-265.

30. Aderhold C, Faber A, Grobschmidt GM, Chakraborty A, Bockmayer A, et al. (2013) Small molecule-based chemotherapeutic approach in p16-positive and -negative HNSCC in vitro. Anticancer Res 33: 5385-5393. 